\title{
Further considerations on in vitro skeletal muscle cell death
}

\author{
Michela Battistelli \\ Sara Salucci \\ Sabrina Burattini \\ Elisabetta Falcieri
}

Department of Earth, Life and Environmental Sciences (DiSTeVA), University Carlo Bo, Urbino, Italy

\section{Corresponding author:}

Michela Battistelli

Department of Earth, Life and Environmental Sci-

ences (DiSTeVA)

Campus Scientifico "Enrico Mattei"

Via Ca' le Suore, 2

61029 Urbino, PU, Italy

E-mail: michela.battistelli@uniurb.it

\section{Summary}

The present review discusses the apoptotic behavior induced by chemical and physical triggers in $\mathrm{C} 2 \mathrm{C} 12$ skeletal muscle cells, comparing myoblast to myotube sensitivity, and investigating it by means of morphological, biochemical and cytofluorimetric analyses.

After all treatments, myotubes, differently from myoblasts, showed a poor sensitivity to cell death. Intriguingly, in cells exposed to staurosporine, etoposide and UVB radiation, apoptotic and normal nuclei within the same fibercould be revealed. The presence of nuclear-dependent "territorial" death domains in the syncytium could explain a delayed cell death of myotubes compared to mononucleated cells. Moreover, autophagic granules abundantly appeared in myotubes after each treatment. Autophagy could protect muscle cell integrity against chemical and physical stimuli, making $\mathrm{C} 2 \mathrm{C} 12$ myotubes, more resistant to cell death induction.

KEY WORDS: autophagy, cell death, chemical triggers, myoblasts, myotubes, physical trigger.

\section{Introduction}

Skeletal muscle tissue has been long considered highly resistant to apoptotic cell death. Nevertheless, apoptosis frequently occurs in skeletal muscle biology, where it has been proposed to have a role in development, both in proliferating myoblast and in postmitotic muscle fiber control. Therefore, its deregulation is involved in a number of muscle disorders ${ }^{1}$, such as several muscle dystrophies ${ }^{2-4}$ as well as disuse and sarcopenia ${ }^{5}$.

Under pathological conditions, muscle fibers are capable of mitochondria-mediated events which can lead to apoptosis, characterized by high expression of bax, bcl-2 and caspases, and DNA fragmentation revealed by means of TUNEL analysis and after gel electrophoresis ${ }^{6-8}$.

Mitochondria play a central role in apoptosis of skeletal muscle, which is a plastic tissue able to change its mitochondrial content or composition in response to chronic alteration in use or disuse ${ }^{9}$. Infact, oxidative damage and other stress conditions alter mitochondrial turnover inducing the apoptotic signalling and proteolysis activation ${ }^{10}$.

The aim of the current work is to compare the apoptotic behavior due to chemical and physical triggers in skeletal muscle, at undifferentiated (myoblasts) and differentiated (myotubes) stage. The latter condition represents a particular experimental model, being syncytia multinucleated, in which individual nuclei have been reported to control definite muscle fiber segments ${ }^{11}$.

To highlight mechanisms underlying muscle cell death a variety of chemical apoptotic triggers have been chosen. C2C12 cells have been exposed for a time of $24 \mathrm{~h}$ to $0.5 \mu \mathrm{M}$ staurosporine, $30 \mu$ Mcisplatin, 0.5 mMhydrogen peroxide $\left(\mathrm{H}_{2} \mathrm{O}_{2}\right)$ and $25 \mu$ Metoposide ${ }^{12}$.

Staurosporineis a PKC inhibitor and a ROS generator too ${ }^{13,14}$.

Cisplatin, forming a spectrum of intra- and interstrand DNA cross-links, leads to DNA damage, followed by transcription and replication ${ }^{15}$.

$\mathrm{H}_{2} \mathrm{O}_{2}$ is a well known oxidative stress inducer increasing levels of ROS 9 .

Etoposide, a topoisomerase II inhibitor, induces DNA double strand breaks, leading to p53 activation and apoptosis ${ }^{16}$

It is also known that various physical agents are possible apoptotic inducers, but, in some cases, their mechanism of action is only partially known.

UV-B radiation (290 and $320 \mathrm{~nm}$ ), represents a relevant environmental danger because of their role in skin aging and cancer ${ }^{17}$, as well as in infections exacerbation $^{18}$. UV-B radiation induces oxidative stress, leading, in most experimental models, to apoptotic death. It generally occurs through the intrinsic pathway, even if the extrinsic one cannot be excluded. UV-B radiation appears also effective on cell systems which are normally apoptosis-resistant, such as muscle cells ${ }^{19,20}$.

Hyperthermia-induced apoptosis $\left(43^{\circ} \mathrm{C}\right.$ for 30 minutes followed by 4 hours of post incubation) is character- 
ized both by its distinctive ultrastructural features and by the occurrence of internucleosomal DNA cleavage ${ }^{21,22}$, as demonstrated by our research group in other cells model ${ }^{23-25}$.

Hypothermia $\left(4^{\circ} \mathrm{C}\right.$ for 4 hours followed by 4 hours of post incubation) in some cell types leads to programmed cell death mediated by reactive oxygen species increase ${ }^{26}$.

Low $\mathrm{pH}$ (5-6, followed by 4 hours of post incubation) usually induces DNA damage. It plays an important role in cell death in various pathophysiological condition, including ischemia and cancer. In addition to cell death, acidic $\mathrm{pH}$ has been suggested to be involved in carcinogenesis $^{27}$.

Skeletal muscle cell sensitivity to different triggers were evaluated by means of several morphological, biochemical and cytofluorimetric analyses.

\section{Cells exposed to chemicals}

Control cells (Fig. 1) revealed a good viability. Myoblasts appeared mononucleated and flat, with an elongated fusiform or star-shaped aspect, one large central nucleus with diffuse chromatin and numerous nucleoli (Fig. 1A). Good organelle preservation, in particular that of mitochondria and RER, can be showed after transmission electron microscopy (TEM) observations. Myotubes appeared confluent, very long, and multinucleated (Fig. 1B) and contain up 100 nuclei. These were centrally placed and had an irregular profile compared to those of myoblasts.

Morphological analyses well demonstrated that all treatments generated a diffuse cell damage.

After staurosporine treatment, $\mathrm{C} 2 \mathrm{C} 12$ cell detachment was observed with rounding and dying myoblasts and strongly modified, particularly thin, myotubes. TEM showed a conspicuous secondary necrosis rate in both cell types. A peculiar behavior of chromatin, which appeared organized in dense ring-shaped nuclear patches in both myoblasts (Fig. 2A) and myotubes (Fig. 2F), was revealed.

In both cell types, a in situ DNA fragmentation, consis- tently absent in the control condition, appeared (Fig. $3 A, F)$. A diffuse and intense TUNEL positivity can be observed in myoblasts (Fig. 3B) and, to a lesser extent, in myotubes, where labelled and normal myonuclei were occasionally detected in the same cell (Fig. 3G).

After cisplatin exposure myoblasts presented a rare apoptotic morphology, with chromatin margination and cytoplasmic alterations. A diffuse vacuolisation with swollen and altered mitochondria also appeared (Fig. 2B). Myotubes appeared resistant to this treatment (Fig. $2 \mathrm{G}$ ), showing, nevertheless, a diffuse autophagic vacuolisation (Fig. 2H). After TUNEL reaction only few labelled nuclei were visible in myoblasts (Fig. $3 \mathrm{C}$ ). On the other hand, myotubes appeared totally negative according to the ultrastructural data, indicating a significant resistance to cisplatin cell death induction (Fig. $3 \mathrm{H}$ ).

A general cell damage was evidenced after $\mathrm{H}_{2} \mathrm{O}_{2}$ treatment. Some cells appeared rounded and detached, while others were apparently well preserved. At TEM, myoblasts (Fig 2C) and myotubes (Fig. 2I) show features of secondary necrosis. Both undifferentiated and differentiated cells exposed to oxidative damage presented a number of altered mitochondria (Fig. 2D) and autophagic vacuoles (Fig. 2L). At confocal microscopy, TUNEL-positive myonuclei were observed more diffusely in myoblasts (Fig. 3D) and, to a lesser extent, in myotubes (Fig. 3I).

After etoposide treatment, some myoblasts appeared detached, rounded and blebbed while myotubes presented only an apparent nuclear disorganization. After TEM observations, the myoblasts displayed initial chromatin margination and condensation (Fig. 2E). Similarly to staurosporine response, within the same myotube, normal nuclei coexisted with apoptotic ones (Fig. 2M).

After TUNEL reaction a diffuse DNA fragmentation could be revealed both in myoblasts (Fig. 3E) and myotubes (Fig. 3L). In particular, the latter presented TUNEL negative and positive myonuclei in the same syncytium (Fig. 3M) confirming the ultrastructural observations, and as previously reported (Fig. 3G).

All experimental conditions have been also analysed by means of cytofluorimetric techniques, by using spe-

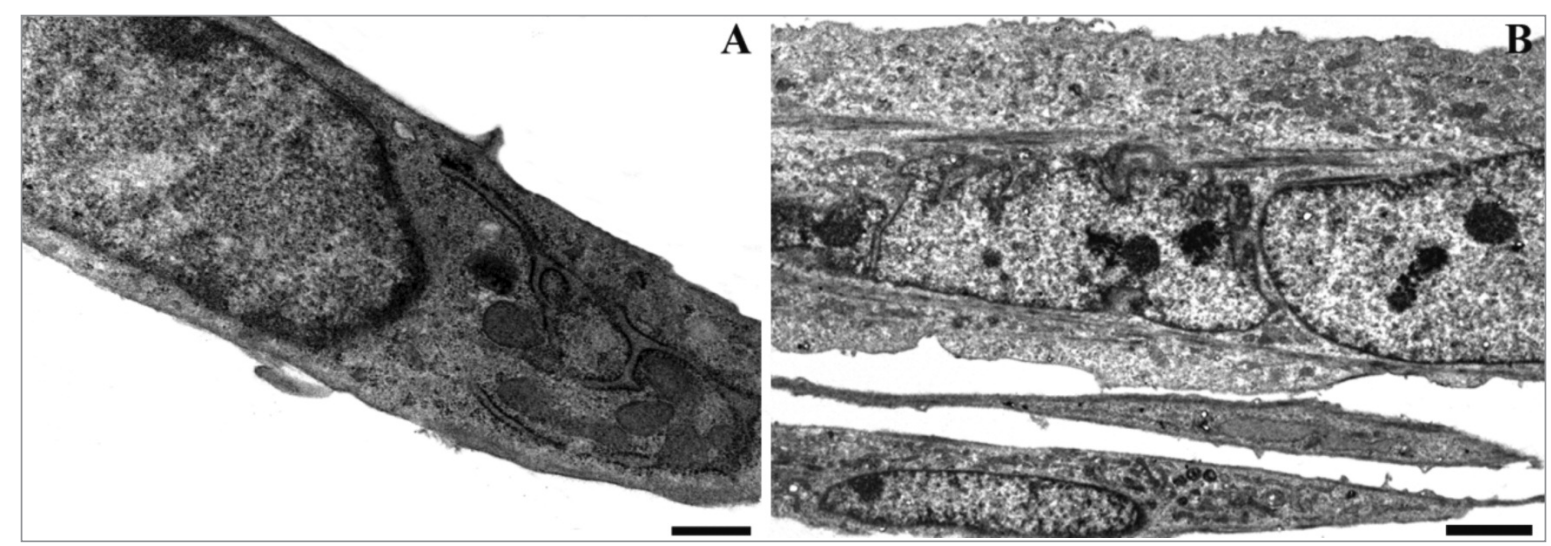

Figure 1. Control myoblasts (A) and myotubes (B) evidence a well preserved morphology at TEM. Scale bars: A, $0.5 \mu$ m; $B$, $2 \mu \mathrm{m}$. 


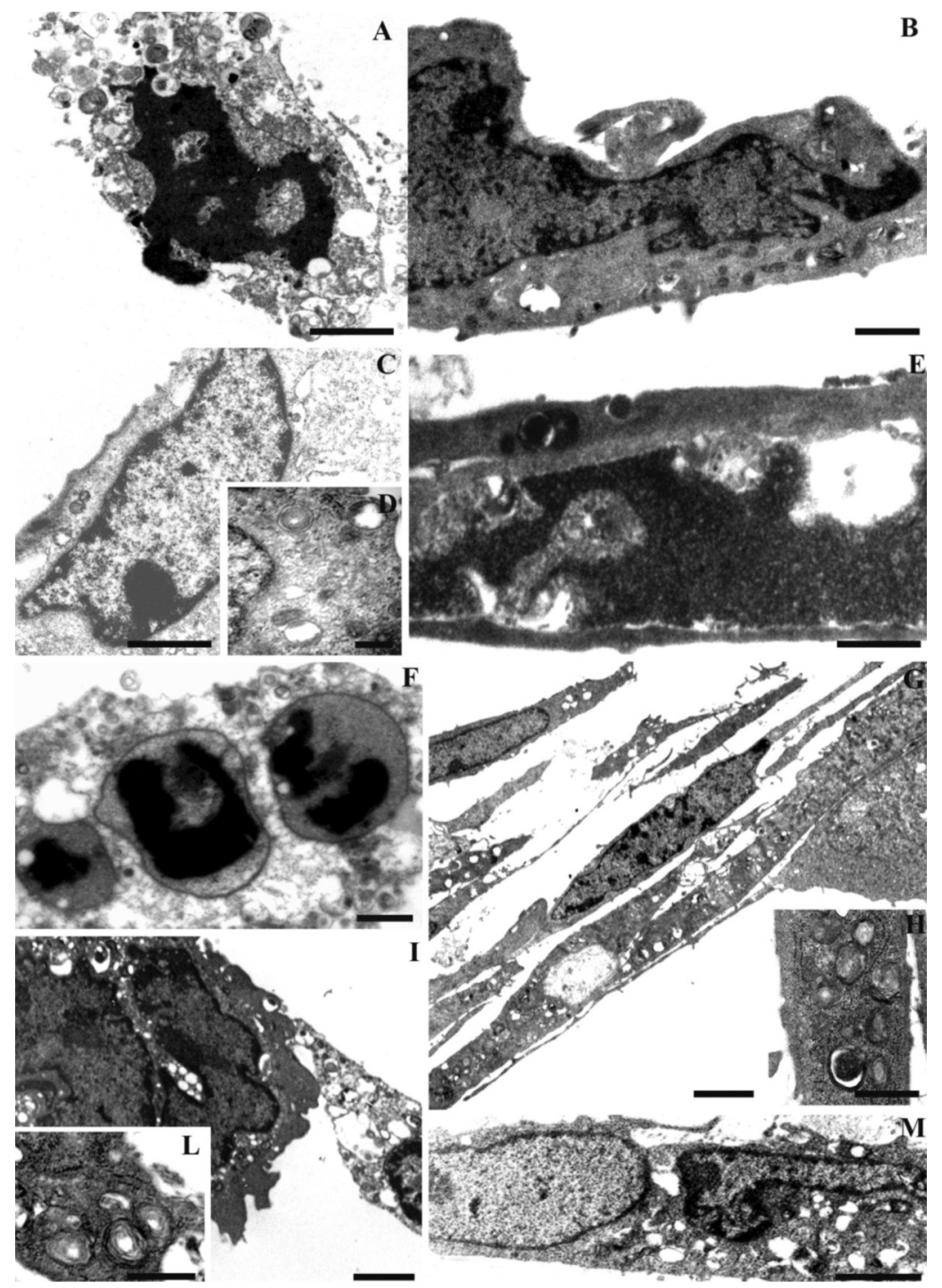

B Figure 2. Cells treated with chemical triggers at TEM.

After staurosporine treatment myoblasts $(A)$ and myotubes $(F)$ show secondary necrosis features. Cisplatin exposure causes an initial chromatin margination in myoblasts (B), an evident cytoplasmic shrinkage and a diffuse autophagic vacuolisation in myotubes $(G, H)$. After hydrogen peroxide administration, cells show occasional necrotic patterns (C, I). In addition, swollen mitochondria and autophagic vacuoles can be observed in both cell types (D, L). Chromatin condensation appears after etoposide $(E$, M). In particular, in myotubes normal nuclei coexist with those presenting an initial chromatin margination (M). Scale bars: A, C, F, G, I, M, $2 \mu \mathrm{m}$; B, $\mathrm{E}, 1 \mu \mathrm{m} ; \mathrm{D}, 250 \mathrm{~nm} ; \mathrm{H}, \mathrm{L}, 500 \mathrm{~nm}$.

cific fluorochromes aimed at evaluating mitochondrial nuclear behavior.

The cardiolipin-sensitive probe 10-nonyl acridine orange (NAO) was used to monitor mitochondrial lipid changes ${ }^{28}$. Cardiolipin peroxidation analysis shows a clear mitochondria impairment in undifferentiated C2C12 cells. In fact, frequencies of events characterized by normal cardiolipin (Fig. $3 \mathrm{~N}$ ) are lowered, in staurosporine, cisplatin, $\mathrm{H}_{2} \mathrm{O}_{2}$ and etoposide-treated myoblasts, indicating the involvement of the mitochondrial pathway and, at the same time, the presence of a certain degree of apoptotic death. On the contrary, in differentiated $\mathrm{C} 2 \mathrm{C} 12$ cells (Fig. 3O), NAO labelling gave almost the same frequencies as control cells, with the exclusion of $\mathrm{H}_{2} \mathrm{O}_{2}$ treated cells $(10 \%$ less than control).

Moreover, we labelled each sample by SYBR GREEN I (Fig. 3P), able to stain nucleic acids with a higher quantum yield when it binds to DNA than when it binds to $\mathrm{RNA}^{29}$. This fluorochrome allowed us to distinguish dif- ferentiated cells with low and high green fluorescence (i.e with low and high DNA content). The differences occurring in green fluorescence were also registered on myoblasts but with a different connotation. In fact, a low DNA content may also be related to apoptotic phenomena, such as dense heterochromatin and DNA cleavage, and linked to a certain shrinkage. On the other hand, in myotubes, cell death was negligible by both ultrastructural and cytometric evaluations and the decrease in SYBR GREEN I fluorescence was not accompanied by cell shrinkage. The Figure $3 \mathrm{P}$ shows that higher percentages of these events were found in etoposide and staurosporine-treated myotubes ${ }^{30}$.

\section{Cell exposed to physical agents}

UV-B irradiated samples revealed deep changes in monolayer organization, as well as significant surface and inner cell ultrastructural alterations. After UV-B 

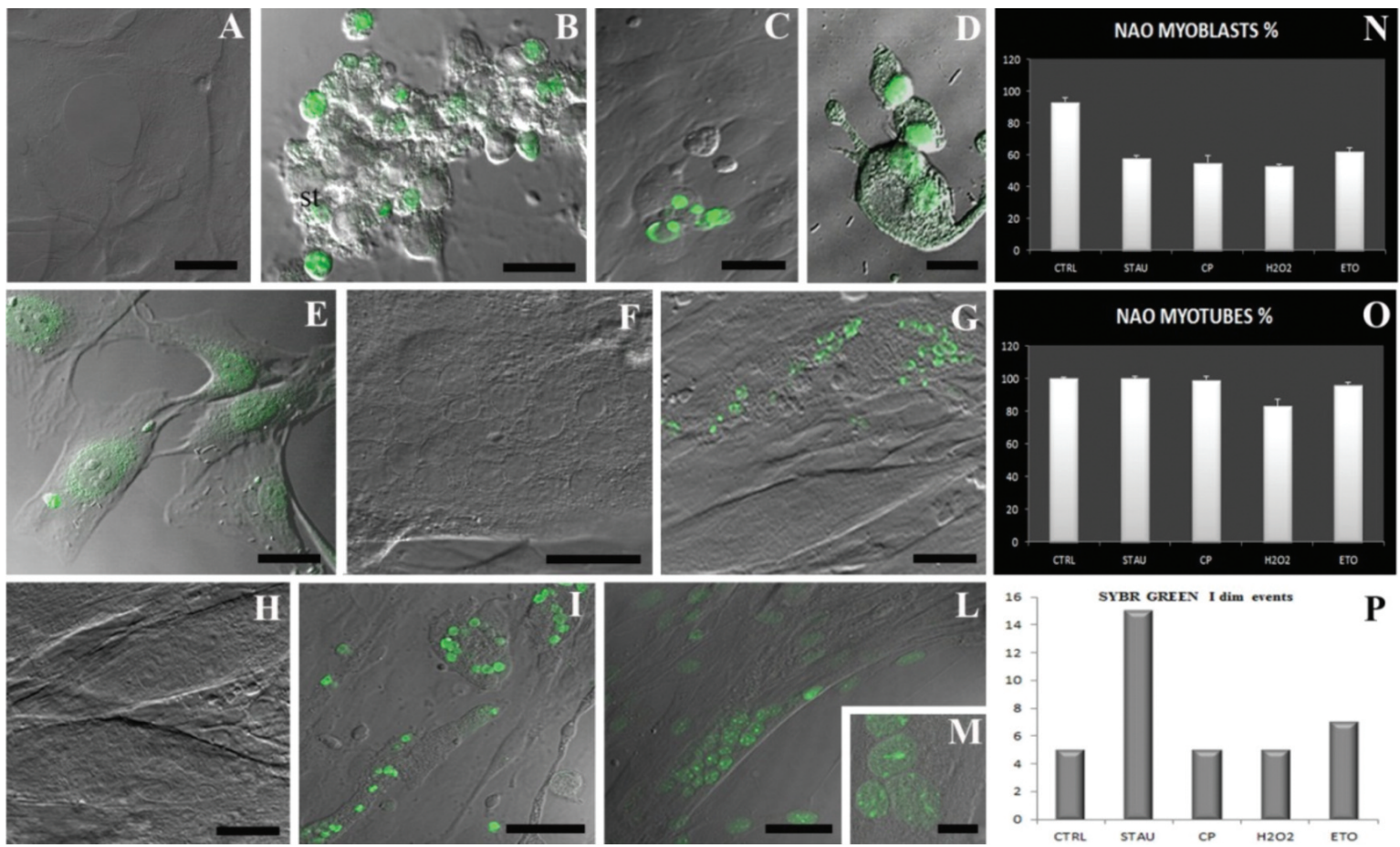

Figure 3. Cells treated with chemical triggers at CLSM and after cytofluorimetric analyses.

TUNEL reaction is negative in control cells $(A, F)$. After staurosporine $(B, G)$, hydrogen peroxide $(D, I)$ and etoposide $(E, L)$ treatment labelled nuclei appear in myoblasts and, in a lesser extent, in myotubes.

In particular, in the same differentiated fiber, exposed to etoposide, fluorescent nuclei coexist in the presence of TUNEL negative nuclei $(\mathrm{M})$. This aspect, has been revealed also through a cytofluorimetric approach. In $\mathrm{P}$, the statistic histogram related to cytometric detection of SYBR GREEN I events, evidences that this peculiar behavior appears in myotubes after etoposide and staurosporine exposure.

After cisplatin, few TUNEL positive nuclei can be observed in myoblasts (C) and they are totally negative in myotubes $(\mathrm{H})$. Mitochondria integrity in control and treated cells has been evaluated through the NAO fluorescence detection. The histograms show the involvement of mitochondrial pathway in undifferentiated $\mathrm{C} 2 \mathrm{C} 12$ cells $(\mathrm{N})$ and the absence of significant mitochondrial impairment in differentiated ones(O).

Scale bars: A, E, $5 \mu \mathrm{m}$; B, C, F, H, L $25 \mu \mathrm{m}$; D, M, $10 \mu \mathrm{m} ; \mathrm{G}$ and I, $50 \mu \mathrm{m}$.

treatment (Fig. 4A), myoblasts showed surface blebs and a few number of cells appeared rounding and detached. The characteristic chromatin margination at the nuclear periphery, sharply separated from the diffuse form, and the presence of nuclear pores were observed at TEM. Interestingly, apoptotic, non apoptotic and necrotic nuclei could be again occasionally observed within the same myotube (Fig. 4E). After TUNEL reaction, cells exposed to UVB show a diffuse number of fluorescent myonuclei (Fig. 5B, G), absent in control myoblasts and myotubes (Fig. 5A, 5F). In this experimental condition, as demonstrated in a previous work $^{19}$, TUNEL positive nuclei coexisted with the normal ones (data not shown).

Cells subjected to acid $\mathrm{pH}$ evidenced strong modifications such as surface blebs and nuclear changes detected both in myoblast and in myotubes. TEM observations evidenced swollen nuclei, revealing a slim film of condensed chromatin (Fig. 4B). In particular, myotubes exposed to low $\mathrm{pH}$ showed features of secondary necrosis, a diffuse cytoplasmic vacuolization (Fig. 4F) and the occasional presence of autophagic vacuoles. After TUNEL reaction labelled nuclei appeared both in myoblasts and myotubes (Fig. 5C, H) After exposure to cold temperature a low number of detached myoblasts and myotubes could be revealed and, at TEM, both cell types showed a well preserved morphology (Fig. 4C) in the presence of autophagic vacuoles (Fig. 4G). A lack of DNA fragmentation has been evidenced after TUNEL reaction in cell subjected to hypothermia (Fig. 5D, I).

Hyperthermia exposure induced cellular shape modifications particularly evident in undifferentiated cells. In addition, surface blebs and necrotic cells were observed at scanning electron microscopy (data not shown). TEM analysis evidenced a certain chromatin condensation (Fig. 4D, H), while a diffuse TUNEL positivity could be observed at CLSM both in undifferentiated and differentiated cells (Fig. 5E, L).

Flow cytometry was used to investigate mitochondrial membrane potential response to UVB-radiation (Fig. 5M). MT red CMXRos analysis was conducted in both control (emptyhistogram) and UVB (filled histogram) conditions. In UVB irradiated cells, histograms showed 


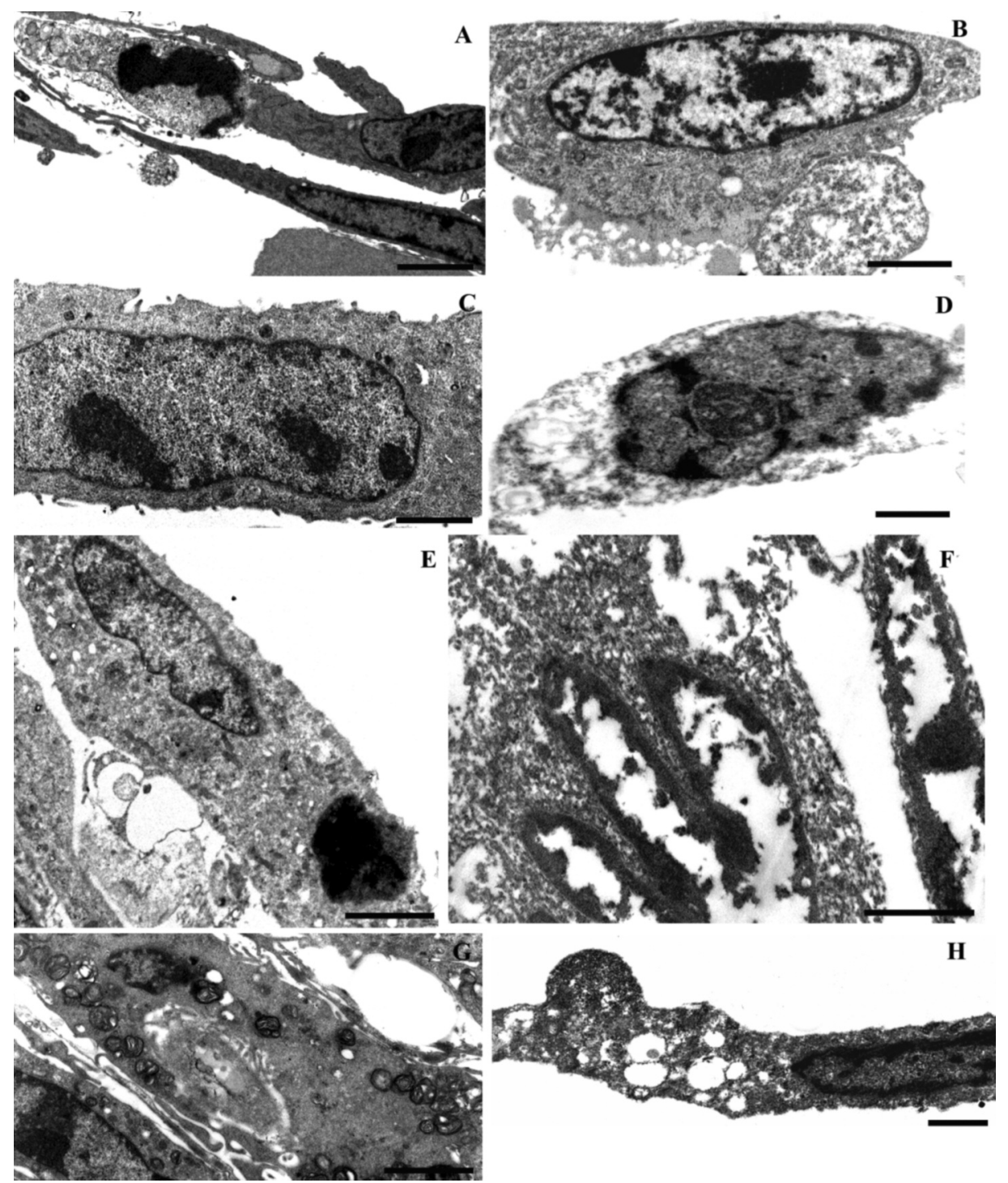

Figure 4. Cells treated with physical triggers at TEM. Apoptotic cells appear after UVB radiation (A, E). In particular, in the differentiated condition normal and apoptotic nuclei in the same fiber can be observed (E). Swollen nuclei (B, F) appear after $\mathrm{pH}$ alteration. Cells exposed to hypothermia maintain a well preserved morphology $(C, G)$, and in myotubes a diffuse number of autophagic vacuoles can be revealed $(G)$. Hyperthermia exposure induces cellular shape modifications, blebbing, cytoplasmic vacuolisation and chromatin margination (D, H). Scale bars: A-G, $2 \mu \mathrm{m} ; \mathrm{H}, 1 \mu \mathrm{m}$.

a decrease in mitochondrion-specific probe fluorescence,corresponding to a reduction in mitochondrial membrane potential. Furthermore, it was possible to observe a different type of fluorescence loss in myoblasts with respect to myotubes: in fact, in the latter a bimodal distribution seems to represent a possible coexistence of collapsed and functional mitochondria.

\section{Conclusion}

In this work, we compared the response of myoblasts and myotubes to the treatment with chemical or physi- cal apoptotic inducers, acting through DNA damage or oxidative stress.

In particular, it focused the attention on myotubes vs myoblasts apoptosis resistance, evaluated through an ultrastructural approach. TEM revealed that chromatin condensation, a characteristic apoptotic feature more diffuse in myoblasts, appeared to be either organized in ring-shaped dense masses under the nuclear membrane, or uniformly condensed to occupy the entire nucleus. Moreover, CLSM observations after TUNEL reaction evidenced that DNA fragmentation occurred in muscle cells and was more evident in myoblasts than myotubes. 


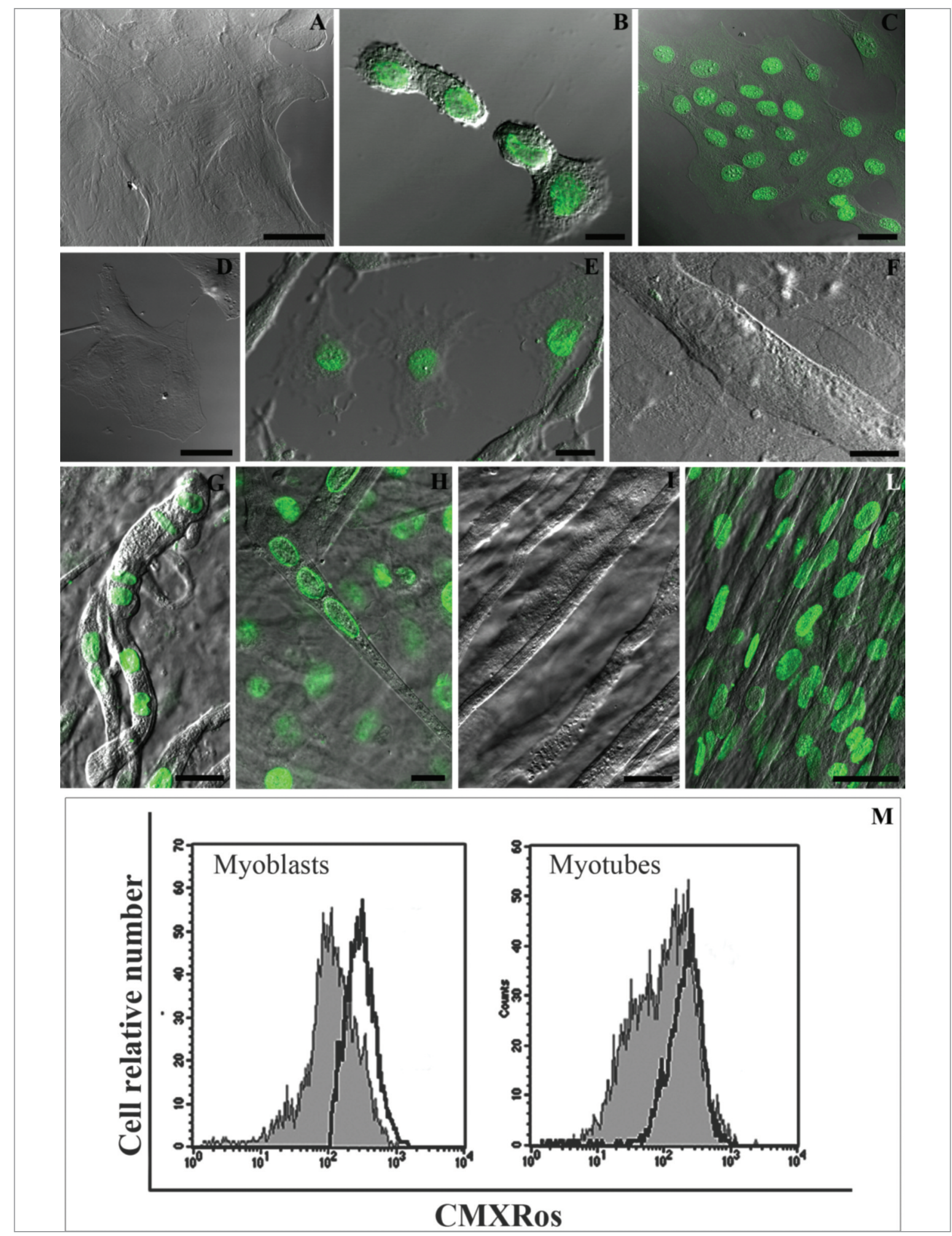

Figure 5. Cells treated with physical triggers at CLSM and after cytofluorimetric analysis. After TUNEL reaction, negative in control cells $(A, F)$, fluorescent nuclei appears after UVB radiation $(B, G), p H$ alteration $(C, H)$ and hyperthermia $(E, L)$. The labelling is absent after hypothermia exposure in both cell types (D, I). Mitochondrial membrane potential has been evaluated by means of CMXRos labelling (M) both in control (emptyhistogram) and UVB irradiated (filled histogram) cells. Histograms show the decrease in mitochondrial membrane potential after UVB irradiation. In particular, in myotubes, it is possible to observe a different type of fluorescence loss with a bimodal distribution, suggesting a possible coexistence of collapsed and functional mitochondria. Scale bars: A,C,D,F,G,H, $10 \mu \mathrm{m} ; \mathrm{B}, \mathrm{E}, 5 \mu \mathrm{m} ; \mathrm{L}, \mathrm{I}, 25 \mu \mathrm{m}$. 
Myotube resistance to apoptosis could be explained considering the cell phenotype: a mononucleated cell is more susceptible to stress signals than a multinucleated syncytium, where each nucleus seems to have an independent domain ${ }^{31}$. In this work, TEM and CLSM demonstrated that by exposing myotubes to etoposide, staurosporine and UVB, apoptotic nuclei coexist with normal nuclei within the same fiber. This aspect has been also detected by means of flow cytometrytrough SYBR GREEN 1, able to distinguish cells with high and low nuclear content. Cells with low nuclear content are those characterized by the coexistence of apoptotic nuclei with normal ones within the same fiber" ${ }^{30}$. This confirms the notion of a "nuclear apoptosis" working to selectively remove the designated myonuclei in the multinucleated syncytium ${ }^{9,32}$.

This phenomenon, even if still considered a controversial point ${ }^{33}$, has been reported in our previous work on UVB-treated myotubes ${ }^{19}$, and has been, recently, reported by other authors ${ }^{34,35}$. Therefore, in mononucleated cells, apoptosis is responsible for nucleus deletion and, later, for the death of the entire cell ${ }^{31}$, while in myotubes apoptosis is limited to one nuclear domain. The deletion of a single nucleus could occur without the death of the entire muscle fiber at the same time, evidencing that a multinucleated cell dies "more slowly"30.

TEM observations revealed a widespread presence of autophagic vacuoles, especially in myotubes, after both chemical and physical treatments. In particular, hypothermia, which is widely used within the sporting context as an immediate relief from muscular trauma pain, has a weak apoptotic effect, in the presence, however, of a diffuse autophagic vacuolisation. This dual effect may be very important in muscle areas subjected to traumatic injury, where death and survival patterns could be active at the same moment. Autophagy, rarely visible in control myoblasts and myotubes, probably play a role in muscle fiber integrity maintenance, as reported by several authors ${ }^{36-38}$. The presence of this mechanism, particularly diffuse in myotube response to chemical and physical triggers, can be considered another element involved in their resistance to cell death.

Moreover, all inducers used and, in particular, $\mathrm{H}_{2} \mathrm{O}_{2}$ and UV-B, are able to increase free radicals and thus oxidative stress, leading to cell death $\mathrm{C} 2 \mathrm{C} 12$ cell line, both at undifferentiated and differentiated condition. Mitochondria activity, analysed by means of flow cytometry, significantly decreased after chemical treatments in myoblasts, whereas in myotubes altered mitochondria appear especially after $\mathrm{H}_{2} \mathrm{O}_{2}$ exposure. Mitochondria dysfunctions have been also observed after UVB exposure; in fact, flow cytometry shows the depolarization of mitochondrial membrane and, as a consequence, the loss of its integrity ${ }^{19}$. Thus, oxidative stress is closely correlated to mitochondrial dysfunctions which appear particularly evident in myoblasts, as also demonstrated by TEM analysis. In fact, in a syncytium cellular antioxidant capability seems to be better preserved, so maintaining a cor- rect ROS level: this may be correlated to myotubes resistance against external stimuli ${ }^{30}$.

In summary, these findings demonstrate a peculiar apoptotic behavior in skeletal muscle cells with a different sensitivity of myoblasts vs myotubes to chemical and physical inducers. The particular organization of myotubes and the appearance of autophagy seem to have a crucial role in muscle response to the different stimuli.

\section{Acknowledgements}

This work was supported by the Ministry of Education, University and Research (PRIN 2009) and Urbino University. The authors are indebted to Dr. Barbara Canonico for flow cytometry, to Dr. Valentina Baldassarri for help in cell cultures, and to Mr. Oliviero Rusciadelli and Mr. Lorenzo Bedini for their skilfull technical assistance in electron microscopy.

\section{References}

1. Tews DS. Role of Apoptosis in Myopathies. Basic Appl Myol 2003; 13:181-190.

2. Rizzuto R, Duchen MR, Pozzan T. Flirting in littlespace: the ER/mitochondria Ca2+ liaison. Sci STKE 2004; 215, re1.

3. Zhao Y, Fedczyna TO, McVicker V, Caliendo J, Li H, Pachman LM. Apoptosis in the skeletal muscle of untreated children with juvenile dermatomyositis: Impact of duration of untreated disease. Clin Immunol 2007; 125:165-172.

4. Girgenrath M, Beermann ML, Vishnudas VK, Homma S, Miller JB. Pathology is alleviated by doxycycline in a laminin-alpha2null model of congenital muscular dystrophy. Ann Neurol 2009 65:47-56.

5. Marzetti E, Calvani R, Cesari M, Buford TW, Lorenzi M, Behnke BJ, Leeuwenburgh C. Mitochondrial dysfunction and sarcopenia of aging: From signaling pathways to clinical trials. Int J Biochem Cell Biol 3013; 45:2288-301.

6. Adhihetty PJ, Hood DA. Mechanisms of Apoptosis in Skeletal Muscle. Basic Appl Myol 2003; 13:171-179.

7. Dupont-Versteegden EE. Apoptosis in skeletal muscle and its relevance to atrophy. World J Gastroenterol 2006; 12 : 7463-7466.

8. Davatz CG, Andreo JC, Rodrigues CA, Rosa Junior GM, Moraes RLH. Apoptosis in denervated skeletal muscle. Int J Morphol 2007; 25:529-536.

9. Siu PM, Wang Y, Always SE. Apoptotic signaling induced by $\mathrm{H} 2 \mathrm{O} 2$ - mediate doxidative stress in differentiated $\mathrm{C} 2 \mathrm{C} 12$ myotubes. Life Sci 2009; 84:468-481.

10. Andrianjafiniony $T$, Dupré-Aucouturier $S$, Letexier $D$, Couchoux H, Desplanches D. Oxidative stress, apoptosis, and proteolysis in skeletal muscle epairafterunloading. Am J Physiol Cell Physiol 2009; 299:C307-315.

11. Adhihetty PJ, Uguccioni G, Leick L, Hidalgo J, Pilegaard H, Hood DA. The role of PGC-1alpha on mitochondrial function and apoptotic susceptibility in muscle. Am J Physiol Cell Physiol 2009; 297:C217-225.

12. Salucci S, Battistelli M, Burattini S, et al. C2C12 myoblast sensitività to different apoptotic chemical triggers. Micron 2010; 41:966-973.

13. Falcieri E, Martelli AM, Bareggi R, Cataldi A, Cocco L. The protein kinase inhibitor staurosporine induces morphological changes typical of apoptosis in Molt-4 cells without concomitant 
DNA fragmentation. Biochem Biophys Res Commun 1993; 193:19-25.

14. Basset O, Boittin FX, Cognard C, Constantin B, Ruegg UT. $\mathrm{Bcl}-2$ over expression prevents calcium over load and subsequent apoptosis in dystrophic myotubes. Biochem J 2006; 395:267-276.

15. Todd RC, Lippard SJ. Inhibition of transcription by platinum antitumor compounds. Metallomics 2009; 1:280-291.

16. Karpinich NO, Tafani M, Rothman RJ, Russo MA, Farber JL. The course of etoposide-induced apoptosis from damage to DNA and p53 activation to mitochondrial release of cytochrome c. J Biol Chem 2002; 277:16547-16552.

17. Lei X, Liu B, Han W, Ming M, He YY. UVB-Induced p21 degradation promotes apoptosis of human keratinocytes. Photochem Photobiol Sci 2010; 9:1640-1648.

18. Srivastava GK, de Larrea GZ. UVB-induced murine bone marrow derived macrophages and apoptosis. Immunol Invest 2008; 37:293-313.

19. D'Emilio A, Biagiotti L, Burattini S, et al. Morphological and biochemical patterns in UVB-induced skeletal muscle cell apoptosis. Histol Histopathol 2010; 25:21-32.

20. Salucci S, Burattini S, Battistelli M, Baldassarri V, Maltarello MC, Falcieri E. Ultraviolet B (UVB) Irradiation-Induced Apoptosis in Various Cell Lineages in Vitro. Int J Mol Sci 2012; 14:532-546

21. Burattini $S$, Ferri $P$, Battistelli $M$, et al. Apoptotic DNA fragmentation can be revealed in situ: An ultrastructural approach. Microsc Res Tech 2009; 72:913-923.

22. Hintzsche H, Riese T, Stopper H. Hyperthermia-induced micronucleus formation in a human keratinocyte cell line. Mutat Res 2012; 738-739:71-74.

23. Luchetti F, Mannello F, Canonico B, Battistelli M, Burattini S, Falcieri E, Papa S. Integrin and cytoskeletonbehaviour in human neuroblastoma cells during hyperthermia-related apoptosis. Apoptosis 2004; 9:635-48.

24. Luchetti F, Burattini S, Ferri P, Papa S, Falcieri E. Actininvolvement in apoptotic chromatin changes of hemopoietic cell sundergoing hyperthermia. Apoptosis 2002; 7:143152.

25. Burattini S, Battistelli M, Luchetti F, Falcieri E. Effects of hyperthermia in human neuroblastoma cells. Ital $\mathrm{J}$ Anat Embryol 2005; 110(2 Suppl 1):49-53.

26. Rauen U, Petrat F, Li T, De Groot H. Hypothermia injury/coldinduced apoptosis-evidence of an increase in chelatable iron causing oxidative injury in spite of low $\mathrm{O} 2-/ \mathrm{H} 2 \mathrm{O} 2$ formation. FASEB J 2000; 14:1953-1964.

27. Knuth ST, Dave H, Peters JR, Fitts RH. Low cell pH depresses peak power in rat skeletal muscle fibres at both 30 degrees $\mathrm{C}$ and 15 degrees $\mathrm{C}$ : implications for musclefatigue. J Physiol 2006; 575:887-899.

28. Luchetti F, Canonico B, Mannello F, et al. Melatonin reduces early changes in intra mitochondrial cardiolipin during apoptosis in U937 cell line. Toxicol In Vitro 2007; 21:293-301.

29. Lebaron $P$, Servais $P$, Agogué $H$, Courties $C$, Joux F. Does the high nucleic acid content of individual bacterial cells allowus to discriminate between active cells and inactive cells in aquatic systems? Appl Environ Microbiol 2001; 67:17751782.

30. Salucci S, Burattini S, Baldassarri V, et al. The peculiar apoptotic behavior of skeletal muscle cells. Histol Histopathol 2013 28:1073-1087.

31. Always SE, Siu PM. Nuclear apoptosis contributes to sarcopenia. Exerc Sport Sci Rev 2008; 36:51-57.

32. Marzetti E, Hwanga JCY, Lees HA, et al. Mitochondrial death effectors: Relevance to sarcopenia and disuse muscle atrophy. Biochim Biophys Acta 2010; 1800:235-244.

33. Bruusgaard JC, Gundersen K. In vivo time-lapse microscopy reveals no loss of murine myonuclei during weeks of muscle atrophy.J Clin Invest 2008; 118:1450-1457.

34. Armand AS, Laziz I, Djeghloul D, et al. Apoptosis-inducing factor regulates skeletal muscle progenitor cell number and muscle phenotype. PLoS One 2011; 6:e27283.

35. Guo BS, Cheung KK, Yeung SS, Zhang BT, Yeung EW. Electrical stimulation influences satellite cell proliferation and apoptosis in unloading-induced muscle atrophy in mice. PLoS One 2012; 7:e30348.

36. Masiero E, Agatea L, Mammucari C, et al. Autophagy is requiredto maintain muscle mass. Cell Metab 2009; 10:507515.

37. Alger HM, Raben N, Pistilli E, et al. The role of tumor necrosis factor-a-related apoptosis-inducing ligand (TRAIL) in mediating autophagy in myositis skeletal muscle: A potential nonimmune mechanism of muscle damage. Arthritis Rheum 2011; 63:3448-3457.

38. Xiao R, Ferry AL, Dupont-Versteegden EE. Cell death-resistance of differentiated myotubes is associated with enhanced anti-apoptotic mechanisms compared to myoblasts. Apoptosis 2011; 16:221-234. 